\title{
Linking Out-of-Body Experience and Self Processing to Mental Own-Body Imagery at the Temporoparietal Junction
}

\author{
Olaf Blanke, ${ }^{1,2,4,5}$ Christine Mohr ${ }^{1,3,4}$ Christoph M. Michel, ${ }^{1,4}$ Alvaro Pascual-Leone, ${ }^{6}$ Peter Brugger, ${ }^{7}$ Margitta Seeck, ${ }^{2}$ \\ Theodor Landis, ${ }^{1,4}$ and Gregor Thut ${ }^{1,4,6}$ \\ ${ }^{1}$ Functional Brain Mapping Laboratory and ${ }^{2}$ Presurgical Epilepsy Unit, Department of Neurology, and ${ }^{3}$ Department of NeuroRehabilitation, University \\ Hospital, 1211 Geneva, Switzerland, ${ }^{4}$ Department of Fundamental Neuroscience, University Medical School, Geneva, Switzerland, ${ }^{5}$ Laboratory of Cognitive \\ Neuroscience, Brain-Mind Institute, Ecole Polytechnique Fédérale de Lausanne, 1015 Lausanne, Switzerland, 'Laboratory for Magnetic Brain Stimulation, \\ Behavioral Neurology Unit, Department of Neurology, Beth Israel Deaconess Medical Center, and Harvard Medical School, Boston, Massachusetts 02215, \\ and ${ }^{7}$ Neuropsychology Unit, Department of Neurology, University Hospital, 8091 Zurich, Switzerland
}

The spatial unity of self and body is challenged by various philosophical considerations and several phenomena, perhaps most notoriously the "out-of-body experience" (OBE) during which one's visual perspective and one's self are experienced to have departed from their habitual position within one's body. Although researchers started examining isolated aspects of the self, the neurocognitive processes of OBEs have not been investigated experimentally to further our understanding of the self. With the use of evoked potential mapping, we show the selective activation of the temporoparietal junction (TPJ) at 330-400 ms after stimulus onset when healthy volunteers imagined themselves in the position and visual perspective that generally are reported by people experiencing spontaneous OBEs. Interference with the TPJ by transcranial magnetic stimulation (TMS) at this time impaired mental transformation of one's own body in healthy volunteers relative to TMS over a control site. No such TMS effect was observed for imagined spatial transformations of external objects, suggesting the selective implication of the TPJ in mental imagery of one's own body. Finally, in an epileptic patient with OBEs originating from the TPJ, we show partial activation of the seizure focus during mental transformations of her body and visual perspective mimicking her OBE perceptions. These results suggest that the TPJ is a crucial structure for the conscious experience of the normal self, mediating spatial unity of self and body, and also suggest that impaired processing at the TPJ may lead to pathological selves such as OBEs.

Key words: self processing; temporoparietal junction; neurology; transcranial magnetic stimulation; event-related potentials; human

\section{Introduction}

Folk psychology postulates a unity between self and body, a "real me" that resides in one's body and is the subject of experience. Cognitive psychologists and philosophers have challenged this assumption and defined several conceptual (e.g., explicit autobiographical memory and beliefs about oneself) and phenomenological aspects of the self [e.g., the feeling of being positioned at a specific location in space (self location); the experience of perceiving the world from one's egocentric visuospatial perspective; the feeling of being the agent of one's actions (agency)] (Neisser, 1988; Gallagher, 2000; Metzinger, 2003). Researchers recently have begun to examine the neural underpinnings of some of these phenomenological aspects of the self, using neuroimaging (Jean-

\footnotetext{
Received July 1, 2004; revised Nov. 12, 2004; accepted Nov. 12, 2004.

This work was supported by the Leenaards Foundation and the Swiss National Science Foundation (Grants 3100-067874.02, 823A-061230, and 3200B0-105867). We thank Thomas Metzinger for his helpful comments on a previous version of this manuscript; Carole Peyrin and Katsuyuki Machii for help in data analysis; and Denis Brunet for his excellent software, Cartool, and his technical assistance in data analysis.

Correspondence should be addressed to Dr. Olaf Blanke, Laboratory of Cognitive Neuroscience, Brain-Mind Institute, Ecole Polytechnique Fédérale de Lausanne, 1015 Lausanne, Switzerland. E-mail: olaf.blanke@epfl.ch or olaf.blanke@hcuge.ch.

DOI:10.1523/JNEUROSCI.2612-04.2005

Copyright $\odot 2005$ Society for Neuroscience $\quad$ 0270-6474/05/250550-08\$15.00/0
}

nerod, 2001; Ruby and Decety, 2001; Decety and Sommerville, 2003; Vogeley and Fink, 2003; Blanke et al., 2004). This has led to the description of many brain areas that mediate different isolated aspects of the self, including prefrontal cortex, anterior cingulate, postcentral gyrus, precuneus, occipito-temporal junction, insula, superior parietal lobule, and temporoparietal junction (TPJ) (Richter et al., 1993; Jeannerod, 2001; Ruby and Decety, 2001; Decety and Sommerville, 2003; Vogeley and Fink, 2003; Blanke et al., 2004). However, it is not known whether there is a key neural locus that mediates several phenomenological aspects of the self. Here we report converging evidence from two studies in normal subjects and a study in an epileptic patient that together provide novel insights about the common neural origin of three essential phenomenological aspects of the self (spatial unity, self location, egocentric visuospatial perspective). This was done by investigating the neurocognitive mechanisms of the so-called out-of-body experience (OBE).

OBEs are defined as experiences in which a person seems to be awake and to see his body and the world from a location outside his physical body. During an OBE the sense of spatial unity between self and body is thus abnormal, because the self is not experienced as residing within the limits of one's body (Blackmore, 1982; Irwin, 1985; Blanke et al., 2004). Moreover, the self 


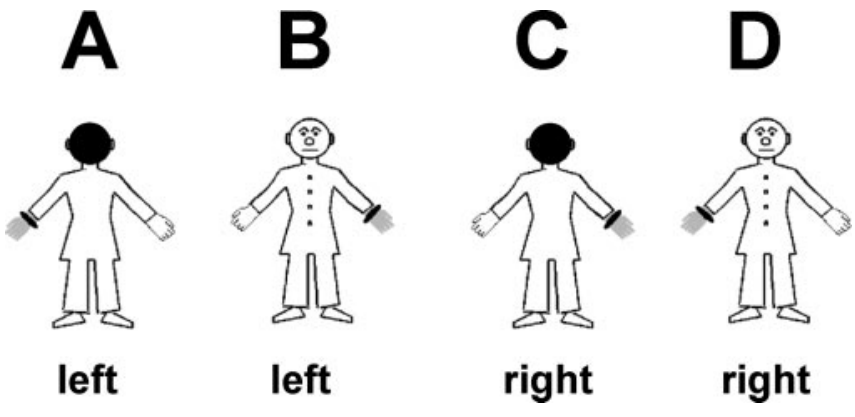

Figure 1. Stimuli. Four different stimuli as used in the OBT task and the lateralization task are shown. Correct responses in the OBT task are indicated below each figure.

seems to be located in a second body that hovers above the physical body (abnormal self location). It is from this elevated visuospatial perspective that the subject has the impression of seeing his body and the world during an OBE (abnormal egocentric visuospatial perspective) (Blackmore, 1982; Irwin, 1985). With respect to experienced unity, self location, and egocentric visuospatial perspective, the OBE thus can be defined as a threefold deviance from the normal self (Metzinger, 2003). Understanding how the brain generates the abnormal self during OBEs is particularly interesting because OBEs not only are found in clinical populations (Brugger et al., 1997; Brugger, 2002; Blanke et al., 2004 ) but appear in $\sim 10 \%$ of the healthy population and are described in the majority of the cultures of the world (Blackmore, 1982; Irwin, 1985). However, there are to date only a few scientific investigations on OBEs, probably because they occur spontaneously, are of short duration, and happen only once or twice in a lifetime (Blackmore, 1982; Irwin, 1985). Investigation of OBEs in neurological and psychiatric disease is not much easier, given their rarity and paroxysmal nature (Brugger et al., 1997; Brugger, 2002; Blanke et al., 2004). However, several authors have linked OBEs to deficient multisensory own-body processing (Blanke et al., 2004) and visuospatial perspective taking (Brugger, 2002). More specifically, some authors proposed that spontaneous OBEs rely on functional mechanisms similar to those used voluntarily during mental imagery with respect to one's visuospatial perspective and body (Brugger, 2002). In addition, we recently have shown that OBEs of neurological origin are related to a dysfunction of the TPJ (Blanke et al., 2002, 2004).

\section{Materials and Methods \\ Evoked potential study}

Participants. Eleven healthy right-handed volunteers (7 male) ages 23-36 years (mean \pm SD, $26.8 \pm 4.0$ years) provided written informed consent to participate in the experiment, the procedures of which were approved by the Ethical Committee of the University Hospital of Geneva (Geneva, Switzerland). Volunteers did not have neurological or psychiatric illnesses, had normal or corrected-to-normal vision, and never had experienced an OBE.

Stimuli and procedure. To test whether OBEs are related to neural mechanisms of mental imagery with respect to one's egocentric visuospatial perspective and one's body, participants performed a modified version of a mental own-body transformation task (OBT task) (Parsons, 1987; Zacks et al., 1999). They were asked to make right-left judgments about a schematic human figure after having imagined themselves to be in the body position of the figure and to have its visuospatial perspective (OBT task) (Fig. 1). The schematic human figure could be facing toward or away from the volunteer. The volunteers' transformation of their own bodies into the body position of the front-facing figures (as compared with the back-facing figures) mentally simulates the body position and the visuospatial perspective that is experienced during OBEs (Blackmore, 1982; Irwin, 1985; Brugger, 2002). Participants were instructed to indi- cate with a button press on a serial response box whether the marked hand of the figure (see below) would be the right or left hand. Front- and back-facing figures had the same outline and differed only in the rendering of the clothing of the figure and the presence of a face (Fig. $1 B, D$, front-facing) or the back of a head (Fig. $1 A, C$, back-facing). The hands of the figure were marked such that one hand appeared as wearing a gray glove and a black ring at the wrist. This indication of side could appear on the right or on the left hand. Stimuli appeared for $200 \mathrm{~ms}$ in the center of the computer screen $\left(5.0 \times 6.1^{\circ}\right.$ of visual angle $)$. The interstimulus interval was $1000 \mathrm{~ms}$. The participants were instructed to respond as fast and precise as possible but always to perform the mental transformation of their body before giving the response. Responses were given with the right hand. Left judgments (Fig. $1 A, B$ ) were indicated by a button press of the index finger and right judgments (Fig. $1 C, D$ ) by a button press of the middle finger. In total, three blocks consisting of a total of 80 stimuli each were performed. In each block each stimulus appeared 20 times in randomized order.

In a control condition (lateralization task) the same visual stimuli were used, but participants decided whether the indicated hand was on the right or the left side of the computer screen (no imagined change in own-body position and visuospatial perspective). This condition was performed to dissociate central mechanisms of OBT from those attributable to the mere perception of the human body (Downing et al., 2001) and right-left decisions. The lateralization task also consisted of three blocks (each with 80 stimuli). Responses were given with the right hand. Again, left judgments were indicated by a button press of the index finger and right judgments by a button press of the middle finger. In six subjects the OBT task (three blocks) was delivered before the lateralization task, whereas in the remaining subjects the tasks were given in reversed order.

Evoked potential mapping. Continuous EEG was acquired from 123 scalp electrodes (Electrical Geodesics, Eugene, OR). The 123 channel evoked potential analysis was based on the examination of the spatial variations of the voltage distribution over time and between conditions, an approach known as evoked potential mapping (Ducommun et al., 2002; Itier and Taylor, 2004). This approach searches for segments of stable map topography that represent functional microstates of the brain during information processing. Evoked potential segments were defined by using a spatial $k$-means cluster analysis to identify the dominant map topographies in the group-averaged evoked potentials across the four experimental conditions over time (Ducommun et al., 2002; Itier and Taylor, 2004). The optimal number of these template maps is determined by a modified cross-validation criterion (Pascual-Marqui et al., 1995). In a second step the presence of a given evoked potential map as identified in the group-averaged data can be verified statistically in the evoked potentials of the individual subjects. This allows us to determine the duration of a given evoked potential map for each condition across subjects. These duration values for a given evoked potential map then can be subjected to statistical analysis. The neural generators for a given evoked potential map were estimated by using a distributed linear inverse solution, based on a local auto-regressive average (LAURA) model (Grave de Peralta et al., 2001). For each subject LAURA was applied at the moment in time when the spatial correlation with a given template map was maximal. We then calculated the mean LAURA solution as the average of all individual source estimations (see Fig. 2E).

\section{Transcranial magnetic stimulation study}

Participants. Seven healthy right-handed volunteers (2 female) ages $19-37$ years (mean \pm SD, $29.6 \pm 5.8$ years) participated in this study. All subjects had given written informed consent before the study. The experiment was approved by the Institutional Review Board of the Beth Israel Deaconess Medical Center (Boston, MA).

Stimuli and procedure. In addition to the OBT task we used a control task that required the participants to transform mentally an external object (see Fig. $3 A$, the letter F). We introduced the letter transformation (LT) task to dissociate mental transformations of one's own body from mental spatial transformations of external objects, similar to previous mental imagery studies (Sirigu and Duhamel, 2001; Tomasino et al., 2003). In contrast to these previous studies, however, we compared mental spatial transformations of the entire body, instead of body parts ( $\mathrm{Si}$ - 
rigu and Duhamel, 2001; Tomasino et al., 2003), with mental transformations of external objects. Importantly, we designed the LT task to match the OBT task regarding the axis of mental transformation and complexity (see below) to be able to examine performance and transcranial magnetic stimulation (TMS) interference in a control task that is characterized by approximately the same reaction times (RTs) as the OBT task. Moreover, inclusion of the LT task extended the control condition that we used in the evoked potential studies, which allowed us to isolate the central mechanisms of OBTs from those of the perception of identical stimuli as well as right-left decisions. The LT task was adapted to the OBT task by replacing the schematic human figures by different views of the letter F. The letter was shown either in unturned manner (canonical position) or in turned manner (as if rotated by $180^{\circ}$ around the vertical axis of the letter) (see Fig. $3 A$ ). As in the OBT task the two stimuli were marked on their right or left side, giving rise to four conditions. The subjects were asked to transform mentally the letter into the canonical position (without an imagined change in their own position and perspective) and, after having done so, to indicate whether the black square was on the right or left of the letter. We used this axial LT task to maintain the same vertical axis of transformation as in the OBT task. Stimuli in the OBT and LT tasks had identical horizontal and vertical dimensions $\left(5.4 \times 6.8^{\circ}\right.$ of visual angle). In addition, the indication of side (right or left) was at the same position in the OBT and LT tasks $\left(2^{\circ}\right.$ of horizontal eccentricity). Moreover, stimuli in both tasks were presented for $1400 \mathrm{~ms}$ duration and were preceded by a fixation cross (with a duration of $1400 \mathrm{~ms}$ ). Finally, responses had to be given as fast and accurately as possible after the onset of the stimuli through a right-hand button press (index finger for left judgments and middle finger for right judgments) in both tasks. Stimuli were presented on a 16 inch Apple monitor driven by a Power Mac computer running PsyScope (Cohen et al., 1993). Subjects were seated comfortably in an armchair in front of a computer screen at a distance of $100 \mathrm{~cm}$ while they were performing the two different mental imagery tasks (OBT, LT). Each task was tested in a separate experimental session.

TMS site and TMS intensity. TMS was applied over the right TPJ as well as over a control site at the intraparietal sulcus (IPS), using a $70 \mathrm{~mm}$ figure-of-eight coil connected to a Magstim Super Rapid Transcranial Magnetic Stimulator (2.2 T maximum field strength; Magstim Company, Dyfed, UK). We targeted the right TPJ because electrical stimulation of this site has been reported to induce OBEs (Blanke et al., 2002). The right IPS was chosen as a control site, because the IPS area was not associated with OBT-related activity (see evoked potential study; also see Fig. $2 E$ ) and because the IPS has been related to mental rotation of external objects by previous neuroimaging studies (Harris et al., 2000; Jordan et al., 2001; Gauthier et al., 2002; Podzebenko et al., 2002) as well as TMS studies (Bestmann et al., 2002; Harris and Miniussi, 2003).

The TMS sites were determined on the basis of anatomical landmarks in each subject, using their high-resolution, T1-weighted magnetic resonance images (MRIs) (see Fig. $3 C$ ). The TPJ site was defined as the junction of the supramarginal, angular, and superior temporal gyrus in approximation of the site where OBEs have been evoked by Blanke et al. (2002). The control TMS site was defined at the IPS. The mean location of the TMS site at the TPJ in Talairach space was at $63 \pm 0.6 /-37 \pm$ $1.9 / 20 \pm 1.2(x / y / z \pm \mathrm{SE})$. In the individual subjects (S) the Talairach coordinates for the TMS site at the TPJ included the following: S1-S7, $65 /-28 / 19 ; 63 /-36 / 18 ; 64 /-38 / 19 ; 65 /-42 / 17 ; 62 /-36 / 21 ; 62 /-34 / 24$; $62 /-42 / 25)$. The mean location of the TMS site at the IPS in Talairach space was at $35 \pm 2.7 /-60 \pm 2.9 / 54 \pm 1.2(x / y / z \pm S E)$. In the individual subjects the Talairach coordinates for the TMS site at the IPS included the following: S1-S7, 41/-60/53; 29/-66/52; 37/-67/51; 27/-67/50; 33/ $-59 / 55 ; 30 /-52 / 57 ; 45 /-50 / 57$. The anatomical determination was done some days before the TMS study on the basis of cortical surface plots constructed from the MRIs. MRIs were coregistered at the day of the experiment with the subject's head position for correct coil positioning, using optical tracking via a frameless stereotactic system (Brainsight, Rogue Research, Montreal, Ontario, Canada). The stereotactic system also was used during the experiment for real-time monitoring of correct coil positioning with respect to stimulation sites via online projection on the brain images (Paus, 1999).
TMS intensity was set to a fixed level for all subjects (75\% of maximum stimulator output). Resting motor threshold (right hand) typically ranges between $\sim 49$ and $77 \%$ maximum stimulator output (mean, $60 \%$ ) in the device used in the present experiment [obtained in a population of healthy subjects that were involved in other studies ( $n=39$; female, $n=$ 24; ages $20-38$ years)].

The two different tasks and the two different TMS sites gave rise to a $2 \times 2$ experimental design. Each combination of task and site was tested in different sessions (total $n=4$ ) for which the order was counterbalanced across subjects over 2 separate days (2 sessions per day). Each subject was tested in all tasks and conditions.

Single-pulse TMS protocol. Single TMS pulses were delivered at 15 different delays after visual stimulus onset. Investigated stimulus-topulse onset asynchronies (SOAs) ranged from 100 to $800 \mathrm{~ms}$ after stimulus onset, with increments of $50 \mathrm{~ms}$. These TMS trials were intermixed with trials without any coil discharge to establish a baseline. For each task and TMS site, each of the four stimuli was presented eight times per SOA or no TMS coil discharge. The order of stimuli, SOAs, and trials with or without TMS was randomized across trials.

Statistical analysis. Subjects' average RTs (of correct responses) in TMS trials were normalized to the corresponding baseline performance of the same condition $(\mathrm{RT}$ value $=\mathrm{RT}$ with $\mathrm{TMS}-\mathrm{RT}$ without $\mathrm{TMS})$. This and similar types of corrections commonly have been used with TMS to take into account individual differences in baseline RTs (Schluter et al., 1999; Walsh et al., 1999; Stewart et al., 2001; Devlin et al., 2003). Here we used this correction to account for general intra-individual changes in (baseline) RTs across the different days of the experiment that might have been induced by factors such as fluctuations of each subject's state, vigilance, or other general processes across days. Such fluctuations would affect the comparisons of interest (TPJ vs IPS stimulation) because the site of stimulation was counterbalanced across days (depending on the order of task $\times$ TMS site combinations; see above).

Furthermore, because our evoked potential study showed OBTrelated activity at $\sim 350 \mathrm{~ms}$ and previous studies revealed that mental transformation of objects takes place between $\sim 400$ and $600 \mathrm{~ms}$ after stimulus onset (Pegna et al., 1997; Milivojevic et al., 2003), we expected TMS pulses to interfere with mental transformations at these latencies and therefore collapsed the data for the SOAs of 100-300, 350-550, and $600-800 \mathrm{~ms}$ (each time window consisting of 5 time bins). These data were subjected to repeated measure ANOVAs, with the factors TMS site (TPJ vs IPS), task (OBT vs LT), stimulus orientation (back-facing/unturned vs front-facing/turned), and SOA (100-300/350-550/600-800 $\mathrm{ms})$. Follow-up comparisons of conditions were performed where appropriate.

\section{Intracranial evoked potential study in epileptic patient}

Patient. To examine the functional relationship among mental OBTs, the TPJ, and OBEs more directly, we were interested to examine whether the brain activity attributable to the voluntary mental transformation of the patient's body into a position and visuospatial perspective that she experienced spontaneously during her OBE is related to the pathological brain activity that induced her OBE. Because clinical details have been described previously (Blanke et al., 2004) (patient 2a), they will be repeated only briefly here. The present patient was a 22 -year-old, righthanded female apprentice suffering from complex partial seizures since the age of 7 years. Her seizures were characterized by a right-sided auditory aura followed by fear, staring, bodily automatisms, and impaired consciousness associated with falling and urinal loss. There were rare secondary generalizations. Family and personal history were unrevealing. Seizure frequency of $>10$ per day could not be reduced by various anticonvulsive treatments, and pharmaco-resistant epilepsy was diagnosed. During noninvasive presurgical epilepsy evaluation [involving long-term video-EEG recordings, three-dimensional magnetic resonance imaging (MRI), positron emission tomography, single photon emission computer tomography, and neurological as well as neuropsychological examination (Blanke et al., 2004)] she experienced an OBE during a complex partial seizure that she described as follows. While lying in bed (and waking up), she had "the feeling of being at the ceiling of the room." She "... had the impression that I was dreaming that I 


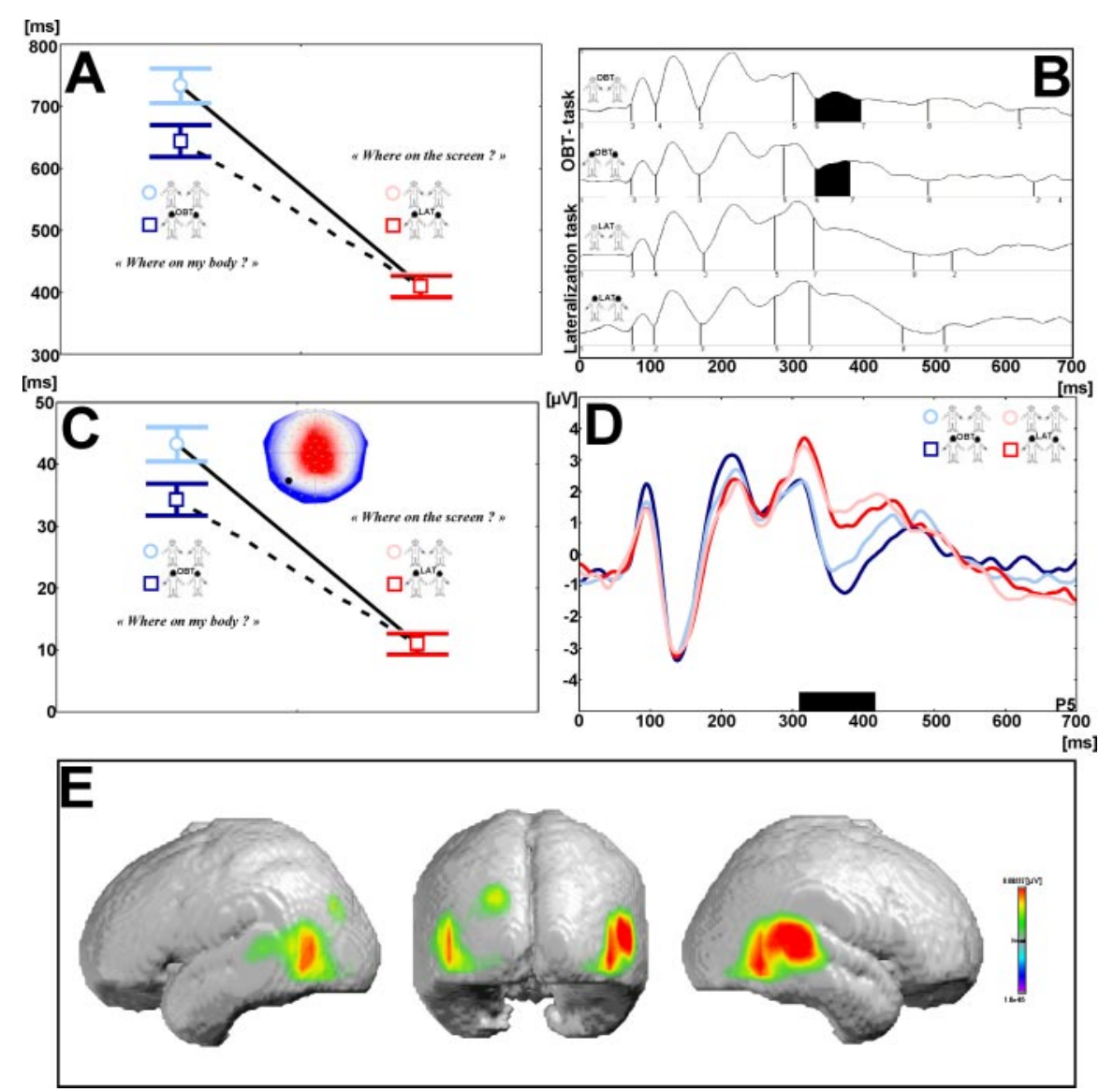

Figure 2. Behavioral and evoked potential data during $O B T$ task and lateralization task (healthy subjects). $A$, Reaction times in the OBT task (blue) and the lateralization task (red). On the left, the RTs (OBT task) are plotted separately for front-facing (light blue) and back-facing figures (dark blue). On the right, the RTs [lateralization task (LAT)] are plotted separately for front-facing (light red) and back-facing figures (dark red). Note the longer RTs for the OBT task. Front-facing figures during the OBT task, but not for the lateralization task, were characterized by longer RTs with respect to back-facing figures. B, Segments of stable map topography in the four experimental conditions under the global field power curve from 0 to $700 \mathrm{~ms}$. Evoked potential segment 6 (segment shown in black) was found from $\approx 330$ to $400 \mathrm{~ms}$ and only in the OBT task. C, Duration of evoked potential segment 6 (the respective map is shown in the right top corner of the figure) for the four experimental conditions for all participants. The duration of evoked potential segment 6 parallels the behavioral differences in the four experimental conditions. D, Evoked potential recorded at electrode P5 in the four experimental conditions showing differential coding between OBT (blue) and lateralization tasks (red) as well as between front-facing (light blue) and back-facing (dark blue) figures during the OBT task. The black bar estimates the time of differential coding between both tasks and is in agreement with the occurrence of evoked potential segment 6 . E, Generators of evoked potential segment 6 (by inverse solution), which were localized bilaterally at the TPJ and predominated at the right TPJ.

would float above (under the ceiling) of the room...." The patient also saw herself as lying in bed, in front view, and she saw "the bed from above." During the postictal phase she presented increased word-finding difficulties. Noninvasive presurgical epilepsy evaluation suggested left temporoparietal seizure onset. To localize the seizure onset zone more precisely and to dissociate it from language cortex, we implanted subdural electrodes. This allowed for definition of the seizure onset zone to a region that included the angular and the posterior superior temporal gyri in the left hemisphere (see Fig. $4 A$ ). Written informed consent was obtained, and the procedures were approved by the Ethical Committee of the University Hospital of Geneva.

Stimuli and task. The OBT stimuli as described for the evoked potential and TMS study were used. For clinical reasons the patient did not perform the lateralization task and LT task. OBT task and intracranial evoked potential (iEP) recordings were performed under identical experimental conditions as the evoked potential study in healthy subjects. The patient performed three blocks of the OBT task (each block consisted of a total of 80 stimuli).

EEG acquisition and analysis. Continuous intracranial EEG was acquired with a Ceegraph IV system (Bio-logic Systems, Mundelein, IL) from implanted subdural grid electrodes (scalp reference, TP10; $200 \mathrm{~Hz}$ digitization; bandpass-filtered, $0.1-70 \mathrm{~Hz}$ ). Subdural grid electrodes were implanted as part of diagnostic investigations and were 3-mm-in-diameter stainless steel electrodes with a center-to-center distance of $0.8 \mathrm{~cm}$. Subdural grid electrodes were MRIcompatible and embedded in a clear silastic sheet (Ad-Tech, Racine, WI). Electrode location was determined by threedimensional MRI of the brain with the implanted electrodes (Lesser et al., 1987; Blanke et al., 1999). A total of 88 subdural grid electrodes was placed over the lateral surface of the left hemisphere covering partly the temporal, parietal, frontal, and occipital lobe. Epochs of EEG from $100 \mathrm{~ms}$ before to $700 \mathrm{~ms}$ after stimulus onset were averaged for each of the two stimulus conditions to calculate the iEP. In addition to the application of an automated artifact criterion of $\pm 80 \mu \mathrm{V}$, the data were inspected visually to reject epochs with epileptic discharges as well as epochs with other transient noise. iEPs were bandpass filtered (1-40 Hz), and we determined the presence, amplitude, and latency of the peaks of the iEPs for all implanted subdural grid electrodes. In a second step we searched whether the cortical sites with prominent iEPs responded differently to front-facing and back-facing figures (Blanke et al., 1999).

\section{Results}

\section{Evoked potential study}

Participants took longer to perform the OBT task $(688.2 \pm 86.2 \mathrm{~ms})$ than the lateralization task $(409.9 \pm 57.7 \mathrm{~ms}$; $\left.F_{(1,10)}=314.31 ; p<0.001\right)$ (Fig. 2A). In addition, RTs were longer for frontfacing figures ( $571.6 \pm 72.8 \mathrm{~ms})$ than for back-facing figures $(526.5 \pm 66.1$ $\left.\mathrm{ms} ; F_{(1,10)}=42.57 ; p<0.001\right)$. The interaction was also significant $\left(F_{(1,10)}=\right.$ 65.44; $p<0.001)$, and the OBT task took longer than the lateralization task if the figure was seen as front-facing as compared with back-facing. Importantly, this front-back difference was specific for the OBT task. This also was reflected in EPs.

Evoked potential mapping of the mean evoked potentials for the four conditions showed that one evoked potential segment with stable voltage topography (Fig. $2 B$, segment 6; time period indicated in black) was longer in the OBT task (36.5 $\pm 16.3 \mathrm{~ms})$ than in the lateralization task $\left(16.5 \pm 16.8 \mathrm{~ms} ; F_{(1,10)}=11.83 ; p<\right.$ 0.003 ) (Fig. 2C). Moreover, the duration of segment 6 was longer for front-facing figures $(30.5 \pm 21.0 \mathrm{~ms})$ than for back-facing figures $\left(22.4 \pm 16.9 \mathrm{~ms} ; F_{(1,10)}=4.55 ; p<0.0456\right)$ (Fig. $\left.2 C\right)$. The interaction was not significant $\left(F_{(1,10)}=0.27 ; p=0.61\right)$. The mean onset of segment 6 was found at $363.8 \pm 38.8 \mathrm{~ms}$. This was also apparent when evoked potentials were analyzed at single temporoparietal electrodes (Fig. 2D, P5), revealing a pronounced evoked potential component (Fig. 2D) that suggests differential coding between the transformation and lateralization task and between the front- and back-facing figures. This differential coding, which classically is observed at a somewhat later time during mental rotation of external objects (Pegna et al., 1997; Milivojevic 
et al., 2003), was found from 300 to 400 $\mathrm{ms}$ in accordance with the occurrence of segment 6 (Fig. 2, compare $B, D$ ). LAURA localized the voltage topography of segment 6 to the TPJ of both hemispheres with a predominant activation in the right hemisphere (mean inverse solution) (Blanke et al., 2002; Zacks et al., 2002). Activation of the TPJ was found in 10 subjects and included both TPJs in four subjects, only the right TPJ in four subjects, and only the left TPJ in two subjects (in one subject activation was found at the right temporo-occipital junction).

\section{TMS study}

To investigate further the implication of the TPJ in processes of mental OBTs, we designed a TMS experiment to examine whether the observed brain activation pattern at the TPJ indeed was required and selective for the mental transformation of one's body and visuospatial perspective or whether it might be related to a more general mental transformation mechanism, i.e., mental transformation of external objects without change in observer's position and visuospatial perspective (Kosslyn, 1994; Pegna et al., 1997; Milivojevic et al., 2003). For this we applied single TMS pulses over the TPJ (test site) and the IPS (control site) in healthy participants (who had never experienced an $\mathrm{OBE}$ ) while performing the OBT task or the LT task (Fig. 3A). We hypothesized (1) that TMS over the TPJ would interfere with the OBT task at those delays when OBT-related activity was found, (2) that interference is selective for TPJ stimulation (relative to stimulation of a control site), and (3) that TPJ interference is task-specific, i.e., not observed for the LT task.

In baseline trials (no TMS applied), RTs were longer for front-facing human figures $(723.7 \pm 58.9 \mathrm{~ms}$; mean \pm SE) and turned letters $(668.9 \pm 52.4$ ms) than for back-facing human figures $(552.1 \pm 38.5 \mathrm{~ms})$ and unturned letters (524.6 $\pm 30.1 \mathrm{~ms}$ ) (effect of stimulus orientation, $F_{(1,6)}=24.4 ; p=$ $0.003)$. This suggests that the subjects engaged in mental spatial transformations. In addition, this effect was independent of task (stimulus orientation $\times$ task, $\left.F_{(1,6)}=1.58 ; p=0.26\right)$. Overall, the LT task appeared to be slightly easier than the OBT task, because subjects responded on average 41 ms faster in the LT than in the OBT task. Nevertheless, this effect was not

\section{A Tasks}

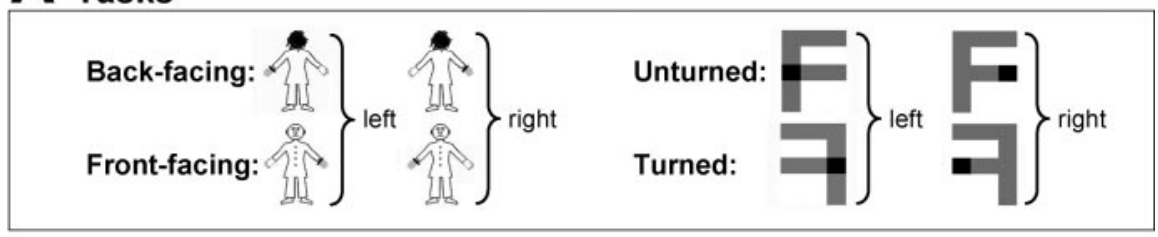

B TMS-effects on RTs per SOA

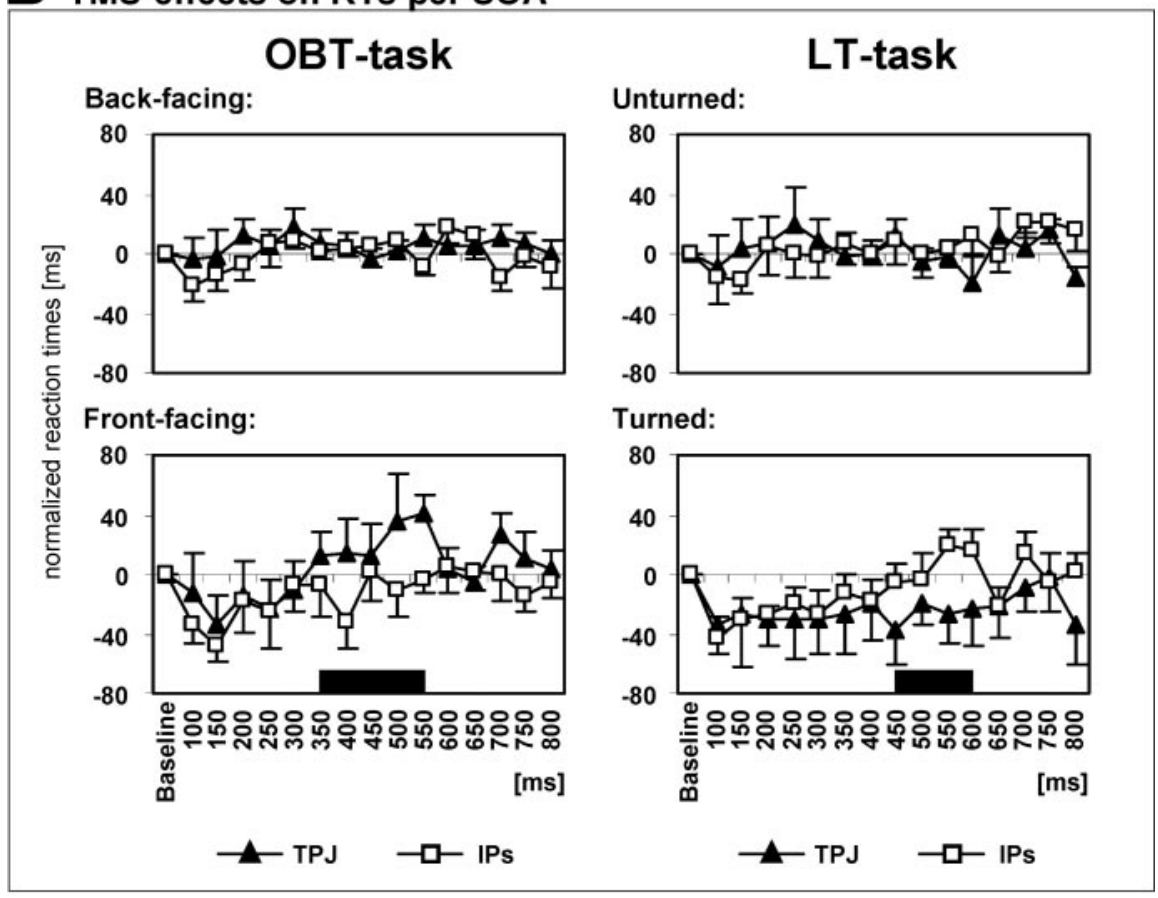

C TMS-sites
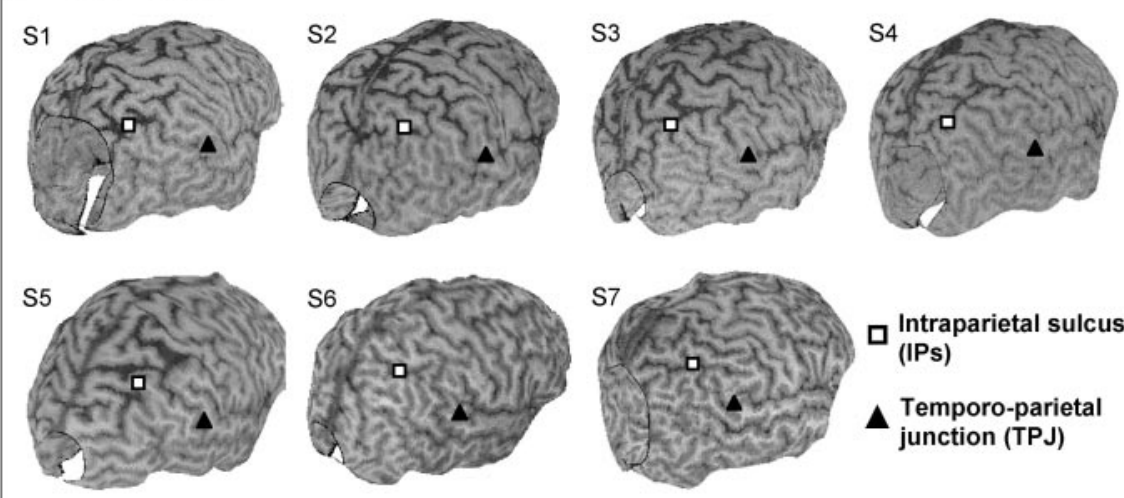

$\square^{\text {Intraparietal sulcus }}$ (IPs)

$\Delta$ Temporo-parietal junction (TPJ)

Figure 3. TMS data during OBT and LT tasks (healthy subjects). $A$, The different stimuli as used in the TMS experiment are shown Correct responses are indicated to the right of each figure. The effects of TMS on the OBT task (human figures) and the LT task (letter F) were tested in a $2 \times 2$ block design ( 2 tasks $\times 2$ TMS sites). B, Normalized RTs for the investigated TMS pulse delays $(100-800 \mathrm{~ms}$ after stimulus onset) for $0 B \mathrm{BT}$ (left) and LT (right) tasks. The top figures plot the RTs for back-facing human figures (OBT task) and unturned letters (LT task) when TMS is applied over either TPJ (filled triangles) or IPS (open squares). The bottom figures show RTs for front-facing figures (OBT task) and turned letters (LT task) for the same TMS conditions. TMS neither of the TPJ nor of the IPS had any effect on performance to back-facing/unturned stimuli (top figures). TMS of the TPJ slowed RTs to front-facing figures (OBT task) relative to IPS stimulation when TMS was applied between 350 and $550 \mathrm{~ms}$ after stimulus onset (bottom left plot). This was concordant with the evoked potential data (time period is indicated by black bar). TMS over IPS, in contrast, increased RTs to turned letters (LT task) relative to TPJ stimulation at TMS pulse delays of $450-600 \mathrm{~ms}$ (bottom right plot). Error bars indicate SES. C, TMS sites at the TPJ and IPS rendered over each subject's cortical surface plot constructed from the individual MRIs. 
significant (baseline trials; nonsignificant trend for task, $F_{(1,6)}=$ 5.62; $p=0.056)$. In addition there were no differences between OBT and LT tasks regarding accuracy [mean error rates $\pm \mathrm{SE}$, $3.1 \pm 0.4 \%$ (OBT task); $2.5 \pm 0.4 \%$ (LT task); paired $t$ test, $t=$ $0.55 ; p=0.59]$.

Importantly, the application of TMS affected RTs in the OBT task (when expressed as change because of TMS in relation to baseline), depending on TMS site, on visual stimulus orientation, and on the delay of the TMS pulse (significant three-way interaction, $F_{(2,12)}=6.70 ; p=0.011$ ) (Fig. $3 B$, left). As hypothesized, TMS led to prolonged RTs for OBTs into front-facing figures (Fig. 3B, bottom left) when applied over TPJ relative to the control site (IPS) but exclusively at specific delays (two-way interaction, TMS site $\times$ SOA, $\left.F_{(2,12)}=5.6 ; p=0.019\right)$ of $350-550 \mathrm{~ms}$ (effect of TMS site, $F_{(1,6)}=10.87 ; p=0.016$ ). No significant effects were observed for back-facing figures (Fig. 3B, top left).

The effect of TPJ stimulation (relative to control stimulation) on OBTs into front-facing figures was task-specific, i.e., it significantly differed from TMS effects on LTs (turned letters) at delays of 350-550 ms (two-way interaction, TMS site $\times$ task, $F_{(1,6)}=$ $12.04 ; p=0.013)$. In contrast to performance in the OBT task (Fig. 3B, bottom left), RTs for LTs were slowed for TMS over IPS (relative to TPJ stimulation) (Fig. $3 B$, bottom right). This effect did not reach significance for delays of 350-550 ms (effect of TMS site, $\left.F_{(1,6)}=2.16 ; p=0.19\right)$ but approached significance for TMS at $450-600 \mathrm{~ms}\left(F_{(1,6)}=5.64 ; p=0.055\right)$. Again, there were no significant effects of TMS on performance to unturned letters (Fig. $3 B$, top right). The TMS data thus suggest that the disruptive effect of TPJ stimulation on OBT with front-facing figures (relative to control stimulation) does not generalize to object-based mental transformations (turned letters) and that OBTs and LTs are likely to be double-dissociated with respect to site and potential timing of TMS interference.

Note that TMS had a general facilitatory effect on RTs with respect to baseline performance (independent of TMS site) for both OBTs (front-facing figures) and LTs (turned letters) (Fig. $3 B$, bottom), which was most pronounced for early TMS pulse delays (reflected in the negative values for normalized RTs). This general facilitatory effect, which also can be observed when a pure auditory signal is presented concomitant to task execution, is most likely to be unspecific to TMS itself and probably is conveyed via mechanisms related to intersensory facilitation attributable to the loud coil click and tap associated with the coil discharge (Terao et al., 1997; Marzi et al., 1998; Sawaki et al., 1999).

\section{Patient study}

In line with the present data in healthy volunteers, the patient responded significantly more slowly for front-facing (757.2 \pm $169.8 \mathrm{~ms})$ than for back-facing figures $(673.3 \pm 120.0 \mathrm{~ms}$; $t$ test; $t=2.845 ; p<0.01)$. This suggests that the mental transformation of her body into the experienced position and visuospatial perspective mimicking her OBE perceptions (front-facing figures) needed more time than the mental transformation of her body into the back-facing figures. This also was reflected in iEPs. The OBT task led to the activation of two brain regions. One region was at the TPJ (Fig. 4A,B) and included the posterior middle and superior temporal plus the anterior angular gyri. The other region was located in the basal temporal lobe, including the fusiform and parahippocampal gyri (Fig. $4 A, C$ ). The activation at the TPJ was reflected by large iEPs at four adjacent electrode sites (mean amplitude, $61.4 \mu \mathrm{V}$; mean latency, $\sim 333 \mathrm{~ms}$ ) (Fig. $4 A, B$ ). The basal temporal activation had an earlier mean latency $(\sim 217$ ms) and a mean iEP amplitude of $76.8 \mu \mathrm{V}$ (Fig. $4 A, C$ ). Crucially,

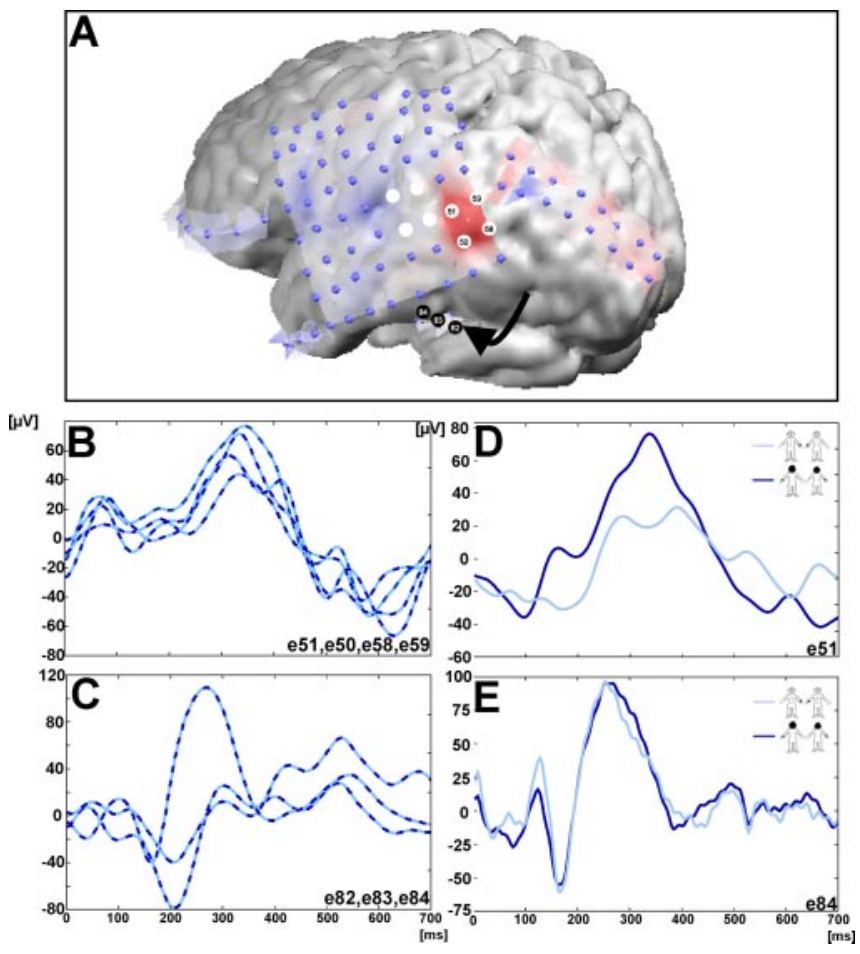

Figure 4. Patient data. A, MRI with the implanted electrodes overlying the lateral convexity of the left hemisphere. The epileptic focus, for which the discharge induced an OBE, is indicated by the eight white electrodes at the TPJ. iEP amplitude (in microvolts) for all implanted electrodes during the $\mathrm{OBT}$ task at $\sim 333 \mathrm{~ms}$ (blue depicts positive values and red depicts negative values). $B$, The most prominent $i$ EPs at this latency were recorded at electrode sites $50,51,58$, and 59 over the TPJ and partly overlapping with the epileptic focus $(A)$. C, Three iEPs during the $O B T$ task, which were recorded in the basal temporal region (indicated by the arrow in $A$ ). Although iEPs at these electrode sites were also prominent, they were recorded at a different latency ( $\sim 270 \mathrm{~ms}$ ). Importantly, as shown in $E$, the amplitude of these basal temporal iEPs did not differ for front-facing (light blue) and back-facing (dark blue) figures, whereas the amplitude of the iEPs at the TPJ did ( $D$ ). The electrode site for each figure is given in the bottom right corner of each panel.

only iEPs at the TPJ showed amplitude differences between frontfacing and back-facing figures (Fig. $4 D, E$ ).

\section{Discussion}

Our evoked potential data show (in healthy subjects who have never experienced an OBE) that mental own-body transformations, which mentally simulate the body position and visuospatial perspective that is experienced spontaneously during OBEs, selectively activate the TPJ at $330-400 \mathrm{~ms}$. In addition, we show that this activation correlates with behavioral measures and phenomenological characteristics of OBEs and found that the activation of the TPJ was longer when subjects imagined themselves as facing themselves (front-facing condition of the OBT task). Finally, the activation at the TPJ is attributable neither to the mere perception of the human body (Downing et al., 2001) nor to right-left decisions. These findings were corroborated by our TMS data by showing that TMS of the TPJ selectively delays the processing of mental own-body transformations with frontfacing figures, but not of external objects such as letters. In addition, TMS effects on mental own-body transformations were found at a similar latency $(350-550 \mathrm{~ms})$ as TPJ activation in the evoked potential study. These findings suggest that the neural mechanisms of own-body transformations can be dissociated temporally and anatomically from the neural mechanisms of the mental rotation of external objects (Kosslyn, 1994; Pegna et al., 
1997; Milivojevic et al., 2003). These latter neural mechanisms classically are observed at a somewhat later time period of 450 $700 \mathrm{~ms}$ in the region of the IPS (Pegna et al., 1997; Harris et al., 2000; Jordan et al., 2001; Gauthier et al., 2002; Podzebenko et al., 2002; Harris and Miniussi, 2003) as also supported by our present findings. In addition to our correlative evoked potential data, these TMS findings are also important because they add evidence for the causal implication (Walsh and Cowey, 2000; Robertson et al., 2003) of the TPJ in a mental OBT task that is linked phenomenologically with OBEs (concerning self location, visuospatial perspective, and spatial unity) (Metzinger, 2003) and frequently used as a means to induce OBEs voluntarily (Blackmore, 1982; Irwin, 1985). Finally, our patient data allow us to link anatomically the mental transformation of one's own body and perspective to neural mechanisms of OBEs by showing that brain activity caused by the voluntary mental transformation of the patient's body into a position and visuospatial perspective that she experienced spontaneously during her OBE led to the activation of the TPJ, but not of other cortical sites in the frontal, parietal, occipital, or temporal lobe. This brain activation was found again at $330-400 \mathrm{~ms}$ and differed for front-facing and back-facing figures concordant with behavioral measures as well as our evoked potential and TMS findings in healthy volunteers. This activation partially overlapped with the epileptic seizure focus, the discharge of which induced her OBE.

Unitary experience between self and body as well as an unambiguous self location and egocentric visuospatial perspective are essential aspects of the self and important mechanisms that allow humans to perceive and interact efficiently within a rapidly changing world (Neisser, 1988; Gallagher, 2000; Metzinger, 2003). Together, our findings demonstrate that these aspects of the self are related to neural activity at the TPJ and that interference with this area can lead to a strikingly deviant self-model, the OBE (Blanke et al., 2002, 2004). Previous neuroimaging studies examining agency (Jeannerod, 2001; Decety and Sommerville, 2003) and visuospatial perspective taking (Ruby and Decety, 2001; Vogeley and Fink, 2003) suggested the involvement of the TPJ in self processing but also found many other cortical areas such as the prefrontal, parietal, or temporal cortex to be involved (Jeannerod, 2001; Ruby and Decety, 2001; Decety and Sommerville, 2003; Vogeley and Fink, 2003). With respect to these previous studies, the present findings suggest that the TPJ might be a key neural locus for self processing within a widely distributed network of cortical areas, although the present investigation focused on the investigation of the role of the TPJ. Whereas single modules of this larger network beyond the TPJ seem to mediate isolated aspects of the self such as agency (Jeannerod, 2001; Decety and Sommerville, 2003), self location (Maguire et al., 1998), and visuospatial perspective (Ruby and Decety, 2001; Vogeley and Fink, 2003), our data suggest that the TPJ mediates the three phenomenological self aspects of visuospatial perspective, self location, and of experienced spatial unity (Metzinger, 2003) that were investigated with the OBT task. In agreement with this, it has been shown that the TPJ and its neighboring structures also are implicated in body-related information processing such as vestibular perception (Lobel et al., 1998; Fasold et al., 2002), multisensory integration (Calvert et al., 2000; Bremmer et al., 2001), as well as the perception of human bodies and body parts (Bonda et al., 1995; Downing et al., 2001; Astafiev et al., 2004). Not only do our results provide psychological and neural evidence for selfmodels that acknowledge the influence of unitary experience, self location, and egocentric perspective (Gallagher, 2000; Metzinger, 2003), but they also suggest that the combined investigation of neurological patients and healthy subjects may be useful in defining the brain structures mediating the normal self, as has been done for other human functions.

In conclusion, the scientific demystification of complex body illusions such as OBEs may be useful in defining the functions and brain structures mediating the normal self and may advance our understanding of their underlying mechanisms, much as previous research into the neural bases of complex body part illusions has demystified phantom limbs (Ramachandran and Hirstein, 1998; Halligan, 2002).

\section{References}

Astafiev SV, Stanley CM, Shulman GL, Corbetta M (2004) Extrastriate body area in human occipital cortex responds to the performance of motor actions. Nat Neurosci 7:542-548.

Bestmann S, Thilo KV, Sauner D, Siebner HR, Rothwell JC (2002) Parietal magnetic stimulation delays visuomotor mental rotation at increased processing demands. NeuroImage 17:1512-1520.

Blackmore S (1982) Beyond the body. An investigation of out-of-body experiences. London: Heinemann.

Blanke O, Morand S, Thut G, Michel CM, Spinelli L, Landis T, Seeck M (1999) Visual activity in the human frontal eye field. NeuroReport 10:925-930.

Blanke O, Ortigue S, Landis T, Seeck M (2002) Inducing illusory own-body perceptions. Nature 419:269-270.

Blanke O, Landis T, Spinelli L, Seeck M (2004) Out-of-body experience and autoscopy of neurological origin. Brain 127:243-258.

Bonda E, Petrides M, FreyS, Evans M (1995) Neural correlates of mental transformations of the body-in-space. Proc Natl Acad Sci USA 92:11180-11184.

Bremmer F, Schlack A, Duhamel JR, Graf W, Fink GR (2001) Space coding in primate posterior parietal cortex. NeuroImage 14:S46-S51.

Brugger P (2002) Reflective mirrors: perspective-taking in autoscopic phenomena. Cogn Neuropsychiatr 7:179-194.

Brugger P, Regard M, Landis T (1997) Illusory reduplification of one's own body: phenomenology and classification of autoscopic phenomena. Cogn Neuropsychiatr 2:19-38.

Calvert GA, Campbell R, Brammer MJ (2000) Evidence from functional magnetic resonance imaging of crossmodal binding in the human heteromodal cortex. Curr Biol 10:649-657.

Cohen JD, MacWhinney B, Flatt M, Provost J (1993) A new graphic interactive environment for designing psychology experiments. Behav Res Methods 24:257-271.

Decety J, Sommerville JA (2003) Shared representations between self and other: a social cognitive neuroscience view. Trends Cogn Sci 7:527-533.

Devlin JT, Matthews PM, Rushworth MF (2003) Semantic processing in the left inferior prefrontal cortex: a combined functional magnetic resonance imaging and transcranial magnetic stimulation study. J Cogn Neurosci 15:71-84.

Downing PE, Jiang Y, Shuman M, Kanwisher N (2001) A cortical area selective for visual processing of the human body. Science 293:2470-2473.

Ducommun CY, Murray MM, Thut G, Bellmann A, Viaud-Delmon I, Clarke S, Michel CM (2002) Segregated processing of auditory motion and auditory location: an ERP mapping study. NeuroImage 16:76-88.

Fasold O, von Brevern M, Kuhberg M, Ploner CJ, Villringer A, Lempert T, Wenzel R (2002) Human vestibular cortex as identified with caloric vestibular stimulation by functional magnetic resonance imaging. NeuroImage 17:1384-1393.

Gallagher S (2000) Philosophical conceptions of the self: implications for cognitive science. Trends Cogn Sci 4:14-21.

Gauthier I, Hayward WG, Tarr MJ, Anderson AW, Skudlarski P, Gore JC (2002) BOLD activity during mental rotation and viewpoint-dependent object recognition. Neuron 34:161-171.

Grave de Peralta R, Gonzalez Andino S, Lantz, G. Michel CM, Landis T (2001) Noninvasive localization of electromagnetic epileptic activity. I. Method descriptions and simulations. Brain Topogr 14:131-137.

Halligan PW (2002) Phantom limbs: the body in mind. Cogn Neuropsychiatr 7:251-268.

Harris IM, Miniussi C (2003) Parietal lobe contribution to mental rotation demonstrated with rTMS. J Cogn Neurosci 15:315-323.

Harris IM, Egan GF, Sonkkila C, Tochon-Danguy HJ, Paxinos G, Watson JD 
(2000) Selective right parietal lobe activation during mental rotation: a parametric PET study. Brain 123:65-73.

Irwin HJ (1985) Flight of mind: a psychological study of the out-of-body experience. Lanham, MD: Scarecrow.

Itier RJ, Taylor MJ (2004) N170 or N1? Spatiotemporal differences between object and face processing using ERPs. Cereb Cortex 14:132-142.

Jeannerod M (2001) Neural simulation of action: a unifying mechanism for motor cognition. NeuroImage 14:S103-S109.

Jordan K, Heinze HJ, Lutz K, Kanowski M, Jancke L (2001) Cortical activations during the mental rotation of different visual objects. NeuroImage 13:143-152.

Kosslyn SM (1994) Image and brain: the resolution of the imagery debate, pp 327-377. Cambridge, MA: MIT.

Lesser RP, Luders H, Klem G, Dinner DS, Morris HH, Hahn JF, Wyllie E (1987) Extraoperative cortical functional localization in patients with epilepsy. J Clin Neurophysiol 4:27-53.

Lobel E, Kleine JF, Bihan DL, Leroy-Willig A, Berthoz A (1998) Functional MRI of galvanic vestibular stimulation. J Neurophysiol 80:2699-2709.

Maguire EA, Burgess N, Donnett JG, Frackowiak RS, Frith CD, O’Keefe J (1998) Knowing where and getting there: a human navigation network. Science 280:921-924.

Marzi CA, Miniussi C, Maravita A, Bertolasi L, Zanette G, Rothwell JC, Sanes JN (1998) Transcranial magnetic stimulation selectively impairs interhemispheric transfer of visuomotor information in humans. Exp Brain Res 118:435-458.

Metzinger T (2003) Being no one. Cambridge, MA: MIT.

Milivojevic B, Johnson BW, Hamm JP, Corballis MC (2003) Non-identical neural mechanisms for two types of mental transformation: event-related potentials during mental rotation and mental paper folding. Neuropsychologia 41:1345-1356.

Neisser U (1988) Five kinds of self-knowledge. Philos Psychol 1:35-59.

Parsons LM (1987) Imagined spatial transformation of one's body. J Exp Psychol Gen 116:172-191.

Pascual-Marqui RD, Michel CM, Lehmann D (1995) Segmentation of brain electrical activity into microstates: model estimation and validation. IEEE Trans Biomed Eng 42:658-665.

Paus T (1999) Imaging the brain before, during, and after transcranial magnetic stimulation. Neuropsychologia 37:219-224.

Pegna A, Khateb A, Spinelli L, Seeck M, Landis T, Michel CM (1997) Unraveling the cerebral dynamics of mental imagery. Hum Brain Mapp 5:410-421.

Podzebenko K, Egan GF, Watson JD (2002) Widespread dorsal stream ac- tivation during a parametric mental rotation task, revealed with functional magnetic resonance imaging. NeuroImage 15:547-558.

Ramachandran VS, Hirstein W (1998) The perception of phantom limbs. Brain 121:1603-1630.

Richter F, Martinez M, Robert M, Bouvier G, Saint-Hilaire JM (1993) Stimulation of human somatosensory cortex: tactile and body displacement perceptions in medial regions. Exp Brain Res 93:173-176.

Robertson EM, Théoret H, Pascual-Leone A (2003) Studies in cognition: the problems solved and created by transcranial magnetic stimulation. J Cogn Neurosci 15:948-960.

Ruby P, Decety J (2001) Effect of subjective perspective taking during simulation of action: a PET investigation of agency. Nat Neurosci 4:546-550.

Sawaki L, Okita T, Fujiwara M, Mizuno K (1999) Specific and non-specific effects of transcranial magnetic stimulation on simple and go/no-go reaction time. Exp Brain Res 127:402-408.

Schluter ND, Rushworth MF, Mills KR, Passingham RE (1999) Signal-, set-, and movement-related activity in the human premotor cortex. Neuropsychologia 37:233-243.

Sirigu A, Duhamel JR (2001) Motor and visual imagery as two complementary but neurally dissociable mental processes. J Cogn Neurosci 13:910-919.

Stewart L, Meyer B, Frith U, Rothwell J (2001) Left posterior BA37 is involved in object recognition: a TMS study. Neuropsychologia 39:1-6.

Terao Y, Ugawa Y, Suzuki M, Sakai K, Hanajima R, Gemba-Shimizu K, Kanazawa I (1997) Shortening of simple reaction time by peripheral electrical and submotor-threshold magnetic cortical stimulation. Exp Brain Res 115:541-545.

Tomasino B, Toraldo A, Rumiati RI (2003) Dissociation between the mental rotation of visual images and motor images in unilateral braindamaged patients. Brain Cogn 51:368-371.

Vogeley K, Fink GR (2003) Neural correlates of the first-personperspective. Trends Cogn Sci 7:38-42.

Walsh V, Cowey A (2000) Transcranial magnetic stimulation and cognitive neuroscience. Nat Neurosci Rev 1:73-79.

Walsh V, Ellison A, Ashbridge E, Cowey A (1999) The role of the parietal cortex in visual attention-hemispheric asymmetries and the effects of learning: a magnetic stimulation study. Neuropsychologia 37:245-251.

Zacks JM, Rypma B, Gabriel J, Tversky B, Glover G (1999) Imagined transformations of bodies: an fMRI investigation. Neuropsychologia 37:1029-1040.

Zacks JM, Ollinger JM, Sheridan MA, Tversky B (2002) A parametric study of mental spatial transformations of bodies. NeuroImage 16:857-872. 